\title{
Polymeric Microfiltration Technology and New Filter Element for Automobile Fuel
}

\author{
Chen Chongjiong ${ }^{\mathrm{a}}$, Chen Xuxu \\ Wenzhou Vocational \& Technical College, Wenzhou, Zhejiang, 325035, China \\ aemail:81383542@qq.com, bemail:185941528@qq.com
}

Keywords: Polymer; Micropore Filter Element; Double-layer Filtration; Pressure Limiting Valve

\begin{abstract}
Paper filter element, widely used in automobile fuel filter, has deficiencies such as short service life and inevitable replacement after blocking. Based on this, the work developed fuel ultra-precision microfiltration technology taking polymer polyethylene as filter material. Meanwhile, it designed filter element of double-layer tube with pressure limiting valve, thus realizing switch of double and single-layer filtration. The work proposed key techniques including polymeric precision microfiltration, filter element manufacturing process, structure design, working principle, main technical characteristics, etc. Results showed that polymeric microfiltration and new filter element technology had advantages including high filtration accuracy, fast filtration speed, good chemical resistance, low cost, micropore regeneration and long service life.
\end{abstract}

\section{Introduction}

Automobile fuel is required to be with high quality and cleanliness. After fuel processing, transportation and storage, fuel consists of impurities such as dust, rust, tar, colloid, moisture, incrustation, etc. The function of automobile fuel filter is to remove the impurities, thus providing the engine with clean and high-quality fuel. If fuel filtration accuracy does not reach the requirements, solid impurity including dust and rust will block fuel supply system, and accelerate mechanical wear of precision components. If the impurities including tar, colloid, moisture, etc. enter the cylinder, they will cause bad burning of combustible mixture and power decline of engine. The work developed fuel ultra-precision microfiltration technology based on polymer filter material. Meanwhile, it designed double-layer polymer filter element with pressure limiting valve.

\section{Situation of automobile fuel filtration technology and filter at home and abroad}

Automobile fuel filter works according to the following principle. Driven by gasoline pump, the fuel is sent to the filter through fuel feed pipe. With increased volume, the flow velocity of the fuel slows down. The water and impurity particles, with larger density than fuel, precipitate on the bottom of filter. While flowing into the filter element along with fuel, the impurities with smaller density are isolated outside, or stuck on the surface of the filter element. After permeating into the filter element through micropore, the fuel drains out of the outlet pipe ${ }^{[1]}$. The filtration efficiency and service life of the filter are determined by filter element. Filter elements of different materials and structures are developed at different times.

At present, micropore paper filter element is widely used in gasoline and diesel filter. Paper filter element has advantages including low price, good passing performance, filtration effect (filtration efficiency $\geqslant 99.6 \%$ ) and easy maintenance. For disposable filter-most widely used filter at home and abroad, the filter element is produced by gluing filter paper cylinder, central network cylinder, top and bottom ends, etc. Filter paper cylinder is treated by phenolic resin to make folding tube. Top and bottom ends are made by steel plate or engineering plastic. Deep processing is required for central network cylinder. Therefore, disposable filter has disadvantages including adhesive failure, short service life, complicated structure and process. Based on adhesive failure, manufacturers make top and bottom ends by phenolic resin. It is difficult to clean paper filter element. Therefore, it should be replaced after blocking. For disposable paper element of fuel filter, the replacement cycle 
is $10,000 \mathrm{~km}$; compulsory replacement cycle $30,000 \mathrm{~km}^{[2]}$.

\section{Development of polymeric precision microfiltration technology}

The work proposed a new ultra-precision filtration-polymeric microfiltration. Its core technology is filtration precision, material formula and manufacturing process of ultra-precision micropore filter element.

\subsection{Designed technique indexes}

Table 1 showed designed technique indexes of polymeric ultra-precision micropore filter element Table 1 Designed technique indexes

\begin{tabular}{cc}
\hline Technical Parameter & Index \\
\hline Micropore Diameter $/ \mu \mathrm{m}$ & $2 \sim 140$ \\
Filtration Precision $/ \mu \mathrm{m}$ & 0.3 \\
Filtration Rate/\% & $\geq 99.99$ \\
Differential Pressure/MPa & $0.2 \sim 0.5$ \\
\hline
\end{tabular}

\subsubsection{Micropore diameter}

Various additives are added into PE powder with ultra high molecular weight. After special manufacturing process, the mixture is calcined at low temperature to produce micropore filter element. Micropore diameter is determined by particle size (or mesh number) of raw materials. Micropre diameter with average diameter of $2 \sim 140 \mu \mathrm{m}$ is made according to different mesh numbers of PE powder.

\subsubsection{Filtration precision}

Filtration precision is the minimum particle size of filtered impurities in fuel. Filtration precision can be calculated by Equation (1) ${ }^{[3]}$.

$$
d_{\mathrm{s}}=d_{\mathrm{m}} \sqrt{\frac{\mu}{\Delta S}} \times \frac{1}{A-B \ln \frac{\bar{\omega}}{\varepsilon}}
$$

where $d_{\mathrm{s}}$ is filtration precision $(\mu \mathrm{m})$; $d_{\mathrm{m}}$ average diameter of undercurrent capillary channels in filter element $(\mu \mathrm{m}) ; \Delta S$ wall thickness of filter element $(\mathrm{mm}) ; \bar{\omega}$ average linear speed of fuel while passing through undercurrent capillary channels $(\mathrm{m} / \mathrm{h}) ; \mu$ fuel viscosity $\left(\mathrm{N} \cdot \mathrm{s} / \mathrm{m}^{2}\right) ; \varepsilon$ average porosity of filter element; A and B are the coefficients of interaction between filtered impurity particles and medium surface.

In Equation (1), $d_{\mathrm{m}}, \mathrm{A}$ and $\mathrm{B}$ are measured by several experiments; $d_{\mathrm{m}}, \Delta S$ and $\bar{\omega}$ adjusted to ensure filtration precision $d_{\mathrm{s}}$.

\subsubsection{Filtration rate}

If filtration precision of filter element is $0.3 \mu \mathrm{m}$, then fuel filtration rate will be larger than $99.99 \%{ }^{[4]}$.

\subsubsection{Differential pressure}

Wall thickness is designed according to the size of micropore filter element. Differential pressure is determined by wall thickness. In general, differential pressure is $0.2 \sim 0.5 \mathrm{MPa}{ }^{[4]}$.

\subsection{Polymeric precision microfiltration technology}

Polymeric ultra-precision microfiltration is one of the most advanced filtration technology. Filter element material is micropore PA and PE rather than PE pipe. Micropores with different diameters assemble to form honeycomb undercurrent channels. These channels have super high filtration precision. (See Figure 1) ${ }^{[5]}$.

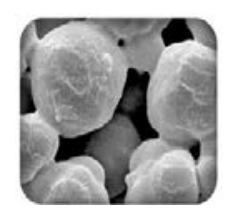

Figure 1 Honeycomb undercurrent channels in micropore filter element

Filtration precision of theoretical calculation is determined by aperture of undercurrent channel. 
There are two filter methods to ensure high precision ${ }^{[6]}$.

(1) In filter medium, the aperture of undercurrent capillary channels does not reach filter precision. Multiple circulation filtration results in the decrease of the aperture and the increase of precision. This method is not suitable for automobile fuel filter.

(2) In filter medium, the aperture of undercurrent capillary channels reaches the filter precision (filtration rate $\geq 99.99 \%$ ). This ideal method has an unsolved core problem. The key of the method is raw material formula and manufacturing process of filter element.

The aperture of undercurrent capillary channel can be controlled based on raw material formula and manufacturing process of filter element. Then the impurities of fuel are filtered once. Polymeric microfiltration medium consists of deep micropore body with certain thickness. Undercurrent capillary channel is bent and alveolate, with high filter capacity and filtration efficiency. Fuel is deeply filtered under flow in three dimensions. Under the same thickness and blocking conditions, the filter can be used for a long time, with slowly increased resistance.

The lower the differential pressure is, the smoother the fuel passes through the filter. Polymer PE micropore filter element has low pressure loss, good passing capacity and fast filter speed (differential pressure=0.2-0.5MPa). Polymer PE filter element has better hardness, strength, compression and shock resistance than paper filter element. In addition, there is no particle and fiber shedding. Therefore, polymer PE microfiltration is better than disposable paper filtration technology.

\subsection{Micropore regeneration}

With the development of micropore filtration technology, filtration method has higher precision, efficiency and lower differential pressure. However, high filtration efficiency increases the block of micropore channel and the difficulty of micropre regeneration.

In fuel filtration process, micro impurities transfer along with the fuel. Some impurities stay, and block micropore undercurrent channel of filter element, thus increasing resistance and thickness of filter cake. If the filtration speed is not suitable for the work, the micropore of filter element will be regenerated by back flushing of compressed gas or back washing of clean water. The filter element can be recycled to improve service life.

\section{Manufacturing process of precision micropore filter element}

The form of micropore undercurrent channel is the key of this technology. The aperture of micropore undercurrent channel is determined by production process. The filter element blank is produced as follows (See Table 2) ${ }^{[7]}$.

Release agent

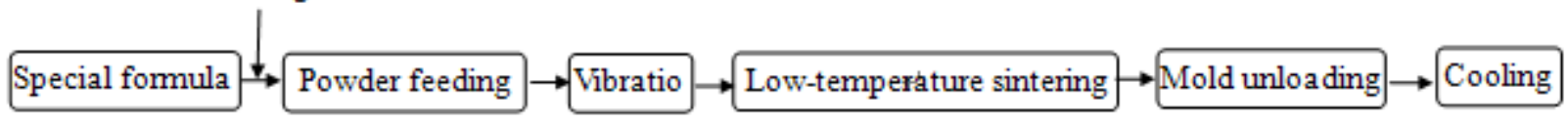

Figure 2 Production process flow of filter element blank

Special formula

(1) Various additives are added to PE powder with super high molecular weight. The mesh number of PE powder with super high molecular weight is selected based on filtration precision.

Powder feeding

(2) After special formula stage, the powders are mixed together, and put in the filter element mold.

Vibration

(3) According to specified vibration frequency, amplitude and time, the powder in the mold is vibrated by linear vibration. Then, surface micropore and internal undercurrent channel meet the requirements of filter precision.

Low-temperature sintering

(4) After vibration, the mixture is sintered in low temperature furnace according to suitable temperature and time.

Mold unloading 
(5) Release agent is brushed on the surface of mold before powder feeding.

(6)Cooling

To avoid deformation, the filter element after mold unloading is cooled at once by water or air cooling.

\section{Technical scheme and structure design of new filter element}

\subsection{Technical scheme}

The filter element consists of polymer ultra-precision micropore with double-layers. Its technical scheme is as follows. Double-layer structure of polymer PE pipe largely improves filtration precision and efficiency of fuel. Long-term filtration results in the increase of filter cake thickness at external surface and the block of internal undercurrent channel, thus increasing the resistance of filter medium. After opening pressure limiting valve, fuel enters ring cavity between external and internal layers. The filtration by internal filter tube extends the service life.

\subsection{Working principle}

The filter element consists of double-layer polymer micropore and pressure limiting valve. The state of pressure limiting valve determines working processes including double and single-layer filtration.

5.2.1 Double-layer filtration while closing pressure limiting valve

If fuel pressure on Steel ball 7 is less than spring force, Steel ball 7 will keep Valve 4 closed by tight pressure (See Figure 3). Fuel is filtered through double-layer polymer PE micropore pipe. After passing through external Filter pipe 1, most impurities are kept outside of Filter pipe 1. Some fine impurities block undercurrent channel in external Filter pipe 1. Some impurities pass through Filter pipe 1 and stay in Annular cavity 3 between the two layers. While passing through Internal filter pipe 2, the impurities are kept on the outside surface. At last, the fuel with little impurities flows out of Outlet 12 . Filtration precision is largely increased (filtration efficiency $\geq 99.99 \%$ ).

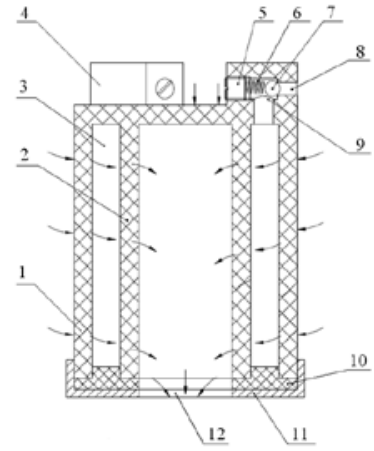

1-External filter pipe;2-Internal filter pipe;3-Annular cavity;4-Pressure limiting valve seat;5-Plug screw;6-Spring; 7-Steel ball;8-Fuel inlet ;9-Fuel outlet of pressure limiting valve;10-Seal ring;11-Gland;12-Fuel outlet of filter element

Figure 3 Closed state diagram of pressure-limiting valve and double-layer filtration

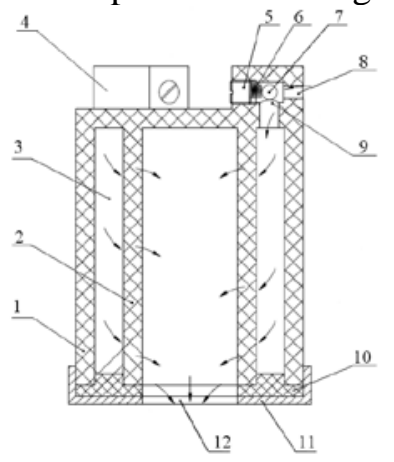

1-External filter pipe;2-Internal filter pipe;3-Annular cavity;4-Pressure limiting valve seat;5-Plug screw;6-Spring; 7-Steel ball;8-Fuel inlet ;9-Fuel outlet of pressure limiting valve;10-Seal ring;11-Gland;12-Fuel outlet of filter element

Figure 4 Open state diagram of pressure-limiting valve and single-layer filtration 
5.2.2 Singer layer filtration while opening pressure limiting valve

Long-term filtration results in the thickening of filter cake formed by impurities outside of External filter pipe 1 or the block of internal undercurrent micropore channels. For this system, the resistance increases, and filter speed slows down. If the fuel pressure on Steel ball 7 is larger than elastic force, then the ball will be jacked to make the pressure limiting valve in the open state (See Figure 4). The fuel enters Annular cavity 3 after passing through fuel inlet and outlet of pressure limiting valve. Internal filter pipe 2 is applied for filtration, thus extending service time for once. Single-layer filtration has lower precision than double-layer filtration. However, polymer microfiltration has a higher precision $(0.3 \mathrm{~mm})$ than paper filtration, thus meeting the requirements of automobile fuel supply system.

\subsection{Filter element structure}

The filter element consists of sub-rigid double-layer micropore filter element, pressure limiting valve, seal ring and gland (See Figure 5).

5.3.1 Structure design of double-layer micropore filter element

It is a sub-rigid double-layer micropore filter element. Filter element consists of external and internal filter pipes ( 1 and 2 ) as well as annular cavity between them. The hatch of annular cavity is set at one end of gland. At the other end of gland, filter element is closed. Double-layer filtration increases filtration area, precision and efficiency.

\subsubsection{Structure design of pressure limiting valve}

To extend service life, pressure limiting valve is set at the closed end of double-layer micropore filter element. The three pressure limiting valves are uniformly distributed around the circumference based on filtration speed and manufacturing process (See Figure 5(b)). Pressure limiting valve consists of Valve body 4, Plug screw 5, Spring 6 and Steel ball 7. Valve body 4 is integrated with filter element. The step through-hole is set in Valve body 4. Connected with annular cavity 3, the through-hole is small outside and large inside. Spring 6 is set between Plug screw 5 and Steel ball 7. Under the elastic force of Spring 6, Steel ball 7 tightly presses the seat of Valve body 4 for sealing, preventing fuel from passing. The open pressure of pressure limiting valve is determined by the rigidity of Spring 6, and adjusted by Plug screw 5.

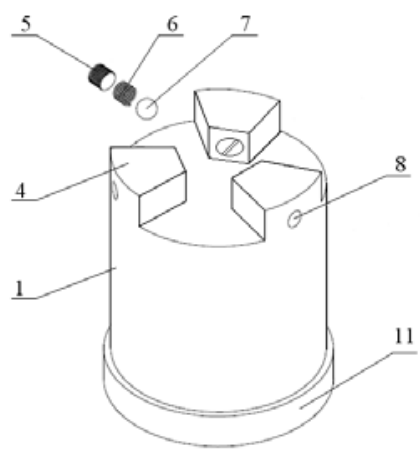

(a) Assembly diagram

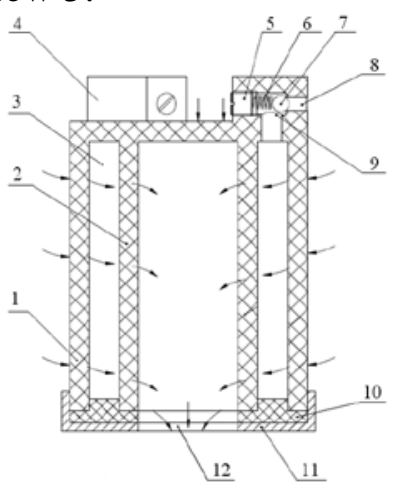

(b) Outline diagram

1-External filter pipe;2-Internal filter pipe;3-Annular cavity;4-Pressure limiting valve seat;5-Plug screw;6-Spring; 7-Steel ball;8-Fuel inlet ;9-Fuel outlet of pressure limiting valve;10-Seal ring;11-Gland;12-Fuel outlet of filter element

Figure 5 Structure diagram of automobile fuel filter element

\section{Main technical characteristics}

\subsection{High filtration precision}

The filter element applies double-layer polymer PE microfiltration to form alveolate undercurrent channel in the filter medium. It has ultra-high filtration precision $(0.3 \mu \mathrm{m})$, with no runout ${ }^{[8]}$.

\subsection{High filtration speed}

For the filter, the lower the differential pressure is, the more smoothly the fuel passes. Polymer 
PE micropore filter element has a differential pressure of $0.2-0.5 \mathrm{MPa}$. It has good passing performance, sufficient fuel supply, high filtration speed and efficiency, thus meeting the requirements of the engine.

\subsection{Good chemical resistance}

Polymer PE micropore filter element has good water and chemical resistance. None of particle and fiber is dropped, and no foreign matter dissolves out, thus ensuring the purity of fuel.

\subsection{Long service life}

The material and structure of filter element are specially designed to prolong the service life.

(1) Double-layer filtration

Long-term filtration results in the thickening of filter cake (formed by impurities outside of External filter pipe 1) or the block of internal undercurrent micropore channels. For this system, the resistance increases, and filter speed slows down, thus causing the increase of pressure outside. Then, Steel ball 7 is jacked to make the pressure limiting valve in the open state. The fuel enters Annular cavity 3 after passing through fuel inlet and outlet of pressure limiting valve. The filtration in Internal pipe 2 extends the service time for once.

(2) Micropore regeneration

Polymer PE micropore filter element applies back flushing of compressive gas or back washing of water to clean the impurities outside of filter element or inside of undercurrent channels. Circulation use of filter element largely extends service life ${ }^{[8]}$.

\section{Conclusions}

Based on polymer PE precision microfiltration, double-layer filter element with pressure limiting valve is designed to improve filtration precision, efficiency and reliability, ensuring stable operation of engine. It has advantages including simple structure, process, low price and long service life. At present, the design has won the national invention patent. Paper filter element has to be replaced after blocking, with short service life. Based on this, the above technology will be widely used to change this situation in automobile parts market. In addition, the technology can also be applied in other filters.

\section{Acknowledgements}

The work was supported by Wenzhou Public Welfare Science \& Technology Project (Project No. C20140058) and Key Task of Scientific Research Project in Wenzhou Vocational \& Technology College (Project No. WZY2013009).

\section{References}

[1] GuanWenDa. Automobile Structure (The Third Edition) [M], China Machine Press, 2010: 79-101.

[2] Guo Xinhua, Automobile Structure (The Second Edition) [M], Higher Education Press, 2010: 79-101.

[3] Song Xianhong, Song Zhili, Micro-filtration of Ultrafine Powders of Metal and Metal Compound [J], Chemical Equipment Technology, 2012(2): 18-22.

[4] Chen Chongjiong, Song Zhiji, Hu Liangfen, Song Xianhong, Filtration, Washing, Recycling Technology and Equipment of Rare Metal Superfine Powders [J], China Powder Science and Technology, 2014(02): 25-30.

[5] Chen Chongjiong erHinfu, Song Zhiji, Design of the All-in-one Unit for Glyphosate Reacting and Filtrating [J], Chemical Industry and Engineering Progress, 2011(12): 2793-2798.

[6] Zhang Youxin, Zhao Zhongwei, Chen Ailiang, Huo Guangsheng, Application of Filtering and 
Washing of Nickel Powder with the Micropore Filter [J], Nonferrous Metals (Extractive Metallurgy), 2008(5): 47-49.

[7] Linear Vibrator [M], Wenzhou Vocational and Technical College, ZL201410256191.8 [P], 2014-09-10.

[8] Chen Chongjiong, Chen Xuxu and Song Zhiji, Recycling Equipment with Filtering and Washing on Fine Powder of Rare Metal Technological Design [J], Advanced Materials Research, 2013: 670-675. 